Educación Física y Ciencia, vol. 20, nº 1, e045, 2018. ISSN 2314-2561

Universidad Nacional de La Plata.

Facultad de Humanidades y Ciencias de la Educación.

Departamento de Educación Física

\title{
Déficit bilateral de las manifestaciones de la fuerza muscular de las extremidades inferiores en estudiantes universitarios
}

Candia-Luján, Ramón; Carreón Santa Cruz, Karen lleana; Núnez Escudero, Beatriz Anai; De León Fierro, Lidia Guillermina ; Carrasco Legleu, Claudia Esther; Candia-Sosa, Kevin Fernando

Cita sugerida: Candia-Luián, R.; Carreón Santa Cruz, K. I.; Núnez Escudero, B. A.; De León Fierro, L. G.; Carrasco Legleu; C. E. y Candia-Sosa, K. F. (2018). Déficit bilateral de las manifestaciones de la fuerza muscular de las extremidades inferiores en estudiantes universitarios. Educación Física y Ciencia, 20(1), e045. https://doi.org/10.24215/23142561e045 


\section{Déficit bilateral de las manifestaciones de la fuerza muscular de las extremidades inferiores en estudiantes universitarios}

Bilateral deficit of the expressions of muscle strength in lower limbs of college students

Ramón Candia-Luján

Facultad de Ciencias de la Cultura Fisica, Universidad Autónoma de Chibuahua, México

rcandia@uach.mx

Karen Ileana Carreón Santa Cruz

Facultad de Ciencias de la Cultura Fisica, Universidad Autónoma de Chibuabua, México kcarreon@uach.mx

Beatriz Anai Núnez Escudero

Facultad de Ciencias de la Cultura Física, Universidad Autónoma de Chibuahua, México bnunez@uach.mx

Lidia Guillermina De León Fierro

Facultad de Ciencias de la Cultura Física, Universidad Autónoma de Chihuabua, México gdeleon@uach.mx

Claudia Esther Carrasco Legleu

Facultad de Ciencias de la Cultura Física, Universidad Autónoma de Chibuabua, México ccarrasco@uach.mx

Kevin Fernando Candia-Sosa

Facultad de Ciencias de la Cultura Física, Universidad Autónoma de Chibuahua, México kev_fer_17@outlook.com

\section{Resumen:}

El objetivo del estudio fue determinar el déficit bilateral (DBL) de las manifestaciones de la fuerza muscular de los miembros inferiores en estudiantes universitarios. Participaron 16 hombres y 14 mujeres. Se les evaluó la fuerza máxima dinámica, la potencia muscular y la fuerza isométrica, en forma bilateral y unilateral de ambas extremidades inferiores. La suma de la fuerza máxima dinámica unilateral de las extremidades inferiores fue mayor que la fuerza bilateral 132,4 $\pm 38,1 \mathrm{~kg}$ y $114,6 \pm 34,7 \mathrm{~kg}$ respectivamente ( $\mathrm{p} \leq 0.05)$; la potencia muscular fue 481,1 $\pm 182,2 \mathrm{~W}$ en la suma de ambos miembros y 441,5 $\pm 181,6 \mathrm{~W}$ para la bilateral $(\mathrm{p} \leq 0,05)$; la suma de la fuerza isométrica de las extremidades fue mayor con 160,1 $\pm 80,2 \mathrm{~kg}$ que la bilateral que resultó de $145 \pm 75,3 \mathrm{~kg}(\mathrm{p} \leq 0,05)$. El DBL de las manifestaciones de la fuerza muscular está presente en los sujetos evaluados, con independencia del género.

Palabras ClaVe: Fuerza máxima, Potencia muscular, Fuerza isométrica, Unilateral, Dominante.

\section{ABSTRACT:}

The aim of the study was to determine the bilateral deficit (BLD) of the expressions of muscle strength of the lower limbs in college students. 16 men and 14 women participated in the study. Their dynamic maximum strength, muscle power and isometric strength were evaluated unilateral and bilaterally in both lower extremities. The sum of the unilateral limbs maximal strength was greater than the bilateral strength $132,4 \pm 38,1 \mathrm{~kg}$ and $114,6 \pm 34,7 \mathrm{~kg}$ respectively $(\mathrm{p} \leq 0,05)$ muscle power was $481,1 \pm 182,2 \mathrm{~W}$ in the sum of extremities and $441,5 \pm 181,6 \mathrm{w}$ for bilateral $(\mathrm{p} \leq 0,05)$, the sum of isometric strength of the two limbs was higher with $160,1 \pm 80,2 \mathrm{~kg}$ while bilateral with $145 \pm 75,3 \mathrm{~kg}(\mathrm{p} \leq 0,05)$. The DBL of the expressions of muscle strength is present in all the subjects tested, regardless of gender.

KEYWORDS: Maximal strength, Muscular power, Isometric strength, Unilateral, Dominant. 


\section{INTRODUCCIÒN}

El entrenamiento de la fuerza ha cobrado gran importancia en los últimos años, no solo en deportes que dependen exclusivamente de ella sino en algunos que son eminentemente aeróbicos, en actividades de la vida diaria, en la estética y en la salud (Nazário-de-Rezende et al. , 2012). Los ejercicios que se incluyen en el entrenamiento de la fuerza muscular pueden ser diversos y ejecutados ya sea de forma bilateral (con los dos miembros a la vez) o unilateral (con un solo miembro) (Ehlers Botton \& Silveira Pinto, 2012).

Desde 1961 se han llevado a cabo estudios donde se ha comparado la fuerza producida por las extremidades homólogas trabajando simultáneamente (ejemplo sentadilla con ambas piernas) contra la suma de la fuerza generada por los miembros activados de forma independiente (sentadilla con una pierna), se ha que visto que la carga total movilizada es mayor cuando se trabaja de esta última forma. La diferencia a favor de esta última fuerza se denomina déficit bilateral (DBL) (Nijem \& Galpin, 2014). El DBL está presente en las diferentes etapas de la vida (Dunstheimer, Hebestreit, Staschen, Strassburg, \& Jeschke, 2001; Kuruganti \& Seaman, 2006; Veligekas \& Bogdanis, 2013) y es más pronunciado en las extremidades inferiores que en las superiores (Magnus \& Farthing, 2008).

No se conoce la influencia de la edad con relación al DBL ya que mientras autores como Hernández, Nelson-Whalen, Franke \& McLean (2003) no encontraron diferencias cuando compararon el DBL entre jóvenes y adultos mayores, Bearskus, Golhlhofer, Muehlbauer, Cardinale \& Grananche (2015) concluyeron que el DBL se incrementaba con la edad.

Brown, Whitehurst, Gilbert, Findley \& Buchalter (1994) encontraron en su estudio que a mayor velocidad de ejecución del ejercicio es menor el DBL de los sujetos participantes. Resultados contrarios encontraron Dickin \& Too (2006) ya que a mayor velocidad de ejecución de las acciones concéntricas el DBL era mayor.

Ya ha pasado más de medio siglo desde que se publicó el primer estudio acerca de este fenómeno cuya causa supone una limitación neural (Ehlers Botton \& Silveira Pinto, 2012).. Van Dieen, Ogita \& De Haan (2003) concluyen en su estudio que durante un esfuerzo bilateral la actividad de la unidad neural se puede reducir hasta el punto de limitar el rendimiento en actividades de intensidad máxima. Kuruganti, Murphy \& Pardy (2011) atribuyen la coactivación de los músculos antagonistas como la causa del DBL. Bobbert, de Graaf, Jonk \& Casius (2006) explican que el DBL en el salto vertical es causado por la relación fuerza-velocidad y no necesariamente por la reducción de la conducción nerviosa.

Aunque la mayoría de los estudios han encontrado que la fuerza desarrollada por la suma de los miembros activados unilateralmente es mayor que cuando se activan simultáneamente algunos autores reportan lo contrario (Ebben, Flanagan, \& Jensen, 2009) o bien que el fenómeno ocurre únicamente durante actividades explosivas pero no durante la generación de la fuerza máxima (Buckthorpe, Pain, \& Folland, 2013). Por su parte MacDonald, Losier, Chester \& Kuruganti (2014) mencionan que los sujetos de su estudio, nadadores y no atletas, presentaron DBL de las extremidades inferiores pero no de las superiores

Los test incluidos en las investigaciones sobre el DBL han sido tanto dinámicos como estáticos (Jakobi \& Chilibeck, 2001), pero no existen estudios donde se haya evaluado la fuerza máxima, la potencia muscular y la fuerza isométrica máxima en los mismos sujetos. Por lo que el presente estudio tuvo como objetivo determinar el DBL de las manifestaciones de la fuerza muscular de las extremidades inferiores en jóvenes universitarios, a partir de la aplicación de tres pruebas que evaluen dichas manifestaciones de la fuerza. 


\section{Metodología}

\section{Participantes}

Participaron en el estudio 30 estudiantes, 16 hombres y 14 mujeres, de la Facultad de Ciencias de la Cultura Física de la Universidad Autónoma de Chihuahua, todos ellos en buen estado de salud, físicamente activos y sin ninguna lesión osteomuscular en los últimos tres años.

En la tabla 1 se puede observar las características generales de los sujetos. En una muestra por conveniencia, los sujetos fueron invitados en forma individual a participar en el estudio, se explicaron el objetivo del estudio así como los beneficios y riesgos de su participación. Se solicitó su firma en una carta de consentimiento informado, redactada de acuerdo a las normas de la Declaración de Helsinki.

\begin{tabular}{|c|c|c|}
\hline & Mujeres $(n=14)$ & Hombres $(n=16)$ \\
\hline Edad (años) & $21,8 \pm 1,6$ & $23,7 \pm 3,2$ \\
\hline Peso $(\mathrm{kg})$ & $63,2 \pm 11,7$ & $76,8 \pm 13,4^{*}$ \\
\hline Estatura $(\mathrm{cm})$ & $163,5 \pm 7,9$ & $175,9 \pm 7,3^{*}$ \\
\hline
\end{tabular}

Tabla 1. Características generales de los participantes

\section{Instrumentos}

La estatura se determinó con un estadímetro de pared (Seca 0901, Alemania). Para el peso corporal se usó una báscula con capacidad de $140 \mathrm{~kg}$ (BAME-420). La fuerza máxima dinámica fue determinada usando una máquina Smith (LifeFitness, EE.UU.A.); para la potencia se utilizó un transductor de posición lineal o TPL (T-Force, España). La fuerza isométrica máxima se midió con el aparato para sentadilla V (LifeFitness, EE.UU. A.) y una celda de carga tipo $S$ con capacidad para $500 \mathrm{~kg}$ (Celtron, EE.UU.A.). Se identificó la pierna dominante preguntando a cada participante con cuál pierna golpeaba más fuerte el balón, lo que permitió el orden de la medición unilateral.

\section{Procedimiento}

Después de un calentamiento de cinco minutos en cicloergómetro los sujetos realizaron tres minutos de estiramiento. El orden de la evaluación bilateral y unilateral de cada extremidad fue aleatorio, así como la secuencia de la medición de la pierna dominante y no dominante. De esa manera, a la mitad de los sujetos se les midió primero la fuerza bilateral y luego la fuerza unilateral de cada miembro inferior y a la otra mitad en forma inversa. El primer día se llevó a cabo la medición de la fuerza máxima dinámica y un día después la potencia y la fuerza máxima isométrica.

Fuerza máxima dinámica. La fuerza máxima se determinó con el test de una repetición máxima (1-RM) siguiendo el protocolo propuesto por Beachle \& Earle (2008) tanto para la sentadilla bilateral como para la unilateral de cada pierna.

Para medir la fuerza bilateral, se colocó a cada sujeto en la barra de la máquina Smith descargada sobre sus hombros, con los pies separados a la anchura de los mismos, se solicitó a cada sujeto posicionarse de forma natural (Comfort \& Kasim, 2007) un poco por delante de la barra, con la mirada al frente o hacia arriba. Se les pidió que realizaran una sentadilla hasta que las rodillas alcanzaran un ángulo de $90^{\circ}$ manteniendo 
la posición mientras el evaluador colocaba un listón en los rieles de la máquina y pintaba una línea en el piso, como referencia para la ejecución del ejercicio. La primera carga administrada fue el equivalente al 50 $\%$ de su peso corporal, realizando ocho sentadillas como calentamiento específico. Después de cada una de las ejecuciones se mostró una escala de percepción del esfuerzo (OMNI-Res) y dependiendo de su indicación y de la ejecución del ejercicio se realizaron los siguientes incrementos de la carga. La pausa de recueración entre intentos fue de tres minutos. Posteriormente se le pidió que hiciera otra repetición, si pudo hacerla se le incrementó el peso nuevamente hasta que ya no pudiera levantar la carga. La fuerza máxima fue considerada como la mayor carga levantada una sola vez o 1-RM.

En el caso de la medición de la fuerza máxima dinámica unilateral se usó la sentadilla unilateral. Cada participante colocó el pie de la pierna contraria a la que sería evaluada sobre un cajón situado en la parte posterior de la máquina Smith, esto con el fin de evitar la intervención muscular de la pierna (Candia-Luján \& de Paz 2015). Posteriormente se siguió el protocolo ya descrito para determinarla.

Potencia muscular o Fuerza explosiva. Para medir la fuerza explosiva se usó un TPL con una velocidad de muestreo de $1000 \mathrm{~Hz}$, fijado a la barra de la máquina Smith. En la evaluación bilateral, a quince sujetos se les colocó el TPL del lado de la pierna dominante y a los restantes en el lado de la no dominante. Durante la evaluación unilateral el TPL se colocó al lado del correspondiente del miembro medido. La carga usada para evaluar la fuerza explosiva fue del 50\% de 1-RM respectiva de cada participante. Después de marcar las referencias de la posición del sujeto para la ejecución del ejercicio se le pidió ejecutar tres sentadillas continuas, indicándole bajar lentamente y permanecer en esa posición hasta la cuenta de tres para, después de una orden verbal subirlos más rápido posible. El TPL fue conectado a una computadora que registró la potencia generada en cada ejecución. Para el análisis de esta manifestación de la fuerza se tomó el valor máximo de las tres ejecuciones.

Fuerza máxima isométrica. La fuerza máxima isométrica se determinó con un aparato para sentadilla $\mathrm{Vy}$ una celda de carga colocada en la parte posterior y fijada con cadenas en cada uno de los extremos. Los sujetos se colocaron debajo de los salientes del aparato apoyando los hombros, se les pidió bajar la cadera flexionando las rodillas hasta $90^{\circ}$ para marcar el lugar donde iban sus pies y ajustar las cadenas de la celda de carga. Para medir la fuerza isométrica máxima se le pidió a cada participante empujar el aparato hacia arriba con la mayor fuerza posible manteniendo la posición por espacio de cinco segundos. Durante la sentadilla unilateral la pierna no evaluada se mantuvo en el aire. Se realizaron tres intentos por cada medición y se consideró el promedio para su análisis.

\section{Análisis estadístico}

El análisis de los datos se llevó a cabo con el paquete estadístico SPSS (IBM, SPSS Statistics, V. 18.0 Chicago, IL, USA) Los datos descriptivos se muestran como media y desviación estándar $(\mathrm{M} \pm \mathrm{DS})$. Se contrastó la normalidad de los datos con la prueba Shapiro Wilk. Para comparar la suma de la fuerza unilateral y la fuerza bilateral se utilizó la t de Student para muestras relacionadas. La comparación del índice de déficit bilateral entre hombres y mujeres se llevó a cabo con la t de Student para muestras independientes. Se estableció un nivel de significancia de $p \leq 0,05$. La significancia estadística práctica se determinó con el tamaño del efecto (d) de Cohen. Tamaño del efecto mayor a 0,8 fueron considerados altos, entre 0,8 y 0,5 moderado, entre 0,5 y 0,2 bajo y menor a 0,2 trivial. Para determinar el porcentaje de déficit bilateral se utilizó la fórmula propuesta por Howard \& Enoka (1991) donde \% DBL $=(100$ - (bilateral/dominante + no dominante $))$ x 100 . 


\section{Resultados}

En la tabla 2 se puede observar que la suma de la fuerza máxima dinámica de los miembros evaluados con la sentadilla unilateral es un $13 \%$ mayor que la fuerza evaluada con la sentadilla bilateral, el mismo efecto se detectó en el análisis de la potencia (12\% mayor la suma de las evaluaciones unilaterales), e igual para la fuerza isométrica máxima donde las evaluaciones unilaterales de ambas piernas fueron un $11 \%$ más altas que la fuerza bilateral encontrada en esta prueba. Todas las comparaciones con diferencia significativa $(p \leq 0,05)$.

\begin{tabular}{lcccc}
\hline Manifestación & Bilateral & Suma Unilateral & DBL & $\mathrm{d}$ \\
\hline Fuerza máxima dinámica (kg) & $114,6 \pm 34,7$ & $132,4 \pm 38,1^{*}$ & $13 \%$ & 0,49 \\
Potencia muscular (W) & $441,5 \pm 181,6$ & $481,1 \pm 182,2^{*}$ & $12 \%$ & 0,22 \\
Fuerza máxima isométrica (kg) & $145 \pm 75,3$ & $160,1 \pm 80,2^{*}$ & $11 \%$ & 0,19 \\
\hline DBL = Déficit bilateral, d = Tamaño del efecto * $^{*}=$ Mayor que la medición bilateral $(p \leq 0.05)$
\end{tabular}

Tabla 2. Resultados obtenidos de la evaluación de las manifestaciones de fuerza $(\mathrm{n}=30)$

En la tabla 3 se puede apreciar que los hombres presentaron mayores valores de las manifestaciones de la fuerza en comparación con las mujeres.

\begin{tabular}{lccc}
\hline & Mujeres & Hombres & $\mathrm{d}$ \\
\hline FMaxD Bil (kg) & $93,6 \pm 35,7$ & $131,4 \pm 25,5^{*}$ & 1,2 \\
\hline Suma FMaxD (kg) & $105,1 \pm 32,8$ & $153,9 \pm 28,6^{*}$ & 1,6 \\
\hline Potencia Bil (W) & $329,7 \pm 150,2$ & $529,9 \pm 166,9 *$ & 1,3 \\
\hline Suma Pototencia (W) & $359,2 \pm 126,2$ & $569,8 \pm 172,7 *$ & 1,4 \\
\hline FMaxIso Bil (kg) & $102,1 \pm 41,4$ & $180,6 \pm 7,2^{*}$ & 2,6 \\
\hline Suma FMaxIso (kg) & $116,5 \pm 49,3$ & $196,9 \pm 83,4^{*}$ & 1,2 \\
\hline Bil = Bilateral, d = Tamaño del efecto, FMaxD = Fuerza máxima dinámica, FMaxIso = Fuerza \\
máxima isométrica. \\
* = Diferencia significativa $(p \leq 0,01)$ entre los valores mujer y hombre & \\
\end{tabular}

Tabla 3. Comparación de los valores absolutos de las manifestaciones de la fuerza entre mujeres y hombres

Como se puede observar en la tabla 4 el DBL fue prácticamente igual en ambos sexos.

\begin{tabular}{|c|c|c|c|}
\hline & \multicolumn{3}{|c|}{$\%$ Déficit bilateral (DBL) } \\
\hline & Mujeres & Hombres & \\
\hline & $(n=14)$ & $(n=16)$ & $\mathrm{d}$ \\
\hline & & & 0, \\
\hline Fuerza máxima dinámica & $14,2 \pm 3,1$ & $12,3 \pm 2,4$ & 7 \\
\hline & & & 0 , \\
\hline Potencia muscular & $12,4 \pm 5$ & $11,2 \pm 2,5$ & 3 \\
\hline & & & 1 , \\
\hline Fuerza má xima isométrica & $12,3 \pm 1,2$ & $10,7 \pm 3,1$ & 0 \\
\hline $\mathrm{d}=$ Tamaño del efecto & & & \\
\hline
\end{tabular}

Tabla 4. Comparación del Déficit bilateral de las manifetaciones de la fuerza entre mujeres y hombres

Cuando hubo diferencia significativa en la comparación de la ejecución bilateral y la suma unilaterel el tamaño del efecto resultó entre trivial y moderado y alto cuando se compararon los valores de las manifestaciones de fuerza entre mujeres y hombres. 


\section{Discusión}

Fuerza máxima dinámica. En el presente estudio la suma de la fuerza máxima dinámica de las extremidades trabajando unilateralmente fue mayor que cuando fueron activadas simultáneamente. Simao, Lemos, Viveiros, Chaves \& Polito (2003) encontraron un $17 \%$ de diferencia entre la suma unilateral y el trabajo bilateral cuando evaluaron la fuerza máxima de la extensión de rodillas a un grupo de mujeres activas físicamente. Por su parte de Souza Bezerra, da Silva Bezerra, Simao, Dias \& Figuereido (2012) reportan un $7 \%$ de DBL para los extensores de la rodilla y un $15 \%$ para los flexores de un grupo de sujetos que habian tenido una cirugía de recontrucción de ligamento cruzado. Chaves et al. (2004) también encontraron DBL en la extensión de la pierna. Por el contrario Monteiro \& Simao (2006) reportan que los sujetos de su estudio tuvieron mayor carga cuando las extremidades trabajaban simultáneamente, tanto miembros superiores como inferiores, aunque en este estudio utilizaron el protocolo de 10-RM.

Potencia muscular o Fuerza explosiva.La potencia muscular es una de las manifestaciones de la fuerza más determinantes tanto en el deporte (Kawamori \& Haff, 2004) como en el ámbito de la salud (Willardson \& Tudor-Locke, 2005). Cuando se ha evaluado el DBL de esta manifestación de la fuerza regularmente se realiza con saltos y plataformas, midiendo la distancia del salto y la fuerza de reacción al piso. En la presente investigación se usó un TPL que ha sido validado para medir este tipo de fuerza (Cronin, Hing, \& McNair, 2004). El uso de la carga al 50\% de 1-RM se estableció similar a estudios previos que establecen que esta intensidad es cercana a la carga con la que se genera la mayor potencia en la máquina Smith (Candia-Luján \& de-Paz-Fernández, 2015; Siegel, Gilders, Staron, \& Hagerman, 2002).

En el presente estudio, los sujetos mostraron un DBL de $12 \%$, porcentaje por debajo a lo reportado por Bracic, Supej, Peharec, Bacic \& Coh (2010) de 19,1 \pm 7,36 \%, aunque los sujetos de su estudio fueron velocistas de élite. Por su parte Pain (2014) evaluó deportistas de potencia y de resistencia y presentaban DBL, pero el del primer grupo fue mayor. Sin embargo, no en todos los estudios se ha encontrado DBL sino por el contrario los sujetos presentaron una facilitación bilateral lo que indica que la fuerza era mayor durante la ejecución bilateral en comparación con la suma bilateral. Los resultados obtenidos tanto por Ebben et al., (2009) y Veligekas \& Bogdanis (2013) fueron contrarios a los de la presente investigación, en el primero de ellos participaron atletas de pista y campo y en el segundo niños y niñas de 10 a 12 años. En este último estudio los estudiantes hombres presentaron facilitación bilateral mientras que para las mujeres no hubo diferencia entre la ejecución bilateral y la suma unilateral.

Fuerza isométrica. El DBL de la fuerza isométrica fue la menor de las presentadas en todas las manifestaciones de la fuerza evaluadas durante esta investigación $(11 \%)$ pero aún así, fueron valores superiores a los reportados por Teixeira, Narciso, Taroco \& Dias (2013) que fue del 7,7 \%, aunque para su estudio los sujetos tenían experiencia en entrenamiento con pesas. Schantz, Moritani, Karlson, Johansson \& Lundh (1989) encontraron resultados parecidos cuando midieron la extensión de rodilla (8-10 \% de DBL). También Matkowski, Martin \& Lepers (2011) reportan DBL de 7,8 \% de los jóvenes activos participantes en su investigación, en tanto Kuruganti \& Seaman (2006) encontraron DBL tanto en extensores como flexores de rodilla de las mujeres y hombres jóvenes y adultos mayores participantes en su estudio. Contrario a los resultados mencionados Howard \& Enoka (1991) reportaron una facilicitación bilateral de 6,2 \% de los extensores de rodilla en levantadores de pesas participantes en su estudio.

Comparación del DBL entre hombres y mujeres. Es bien sabido la diferencia de fuerza existente entre hombres y mujeres, sobre todo en las extremidades superiores (Baechle \& Earle, 2008). La fuerza está determinada principalmente por la sección transversal del músculo (Maughan, Watson, \& Weir, 1983), aunque no necesariamente ésta es la responsable de la diferencia de fuerza entre ambos sexos (MacArdle, Katch, \& Katch, 2010). Sin embargo, también se sabe que los cambios en la fuerza muscular son similares en hombres y mujeres (Cureton, Collins, Hill, \& McElhannon, 1988). Los resultados del presente estudio son congruentes con lo mencionado, ya que a pesar de que los hombres mostraron mayores valores de 
fuerza absolutos en todas las manifestaciones evaluadas, en lo referente al DBL no hubo diferencias con el encontrado en mujeres, lo que indica que el BDL no está influenciado por el sexo.

Por último, debido a la utilización de diferentes aparatos para la medición de las manifestaciones de la fuerza, es dificil comparar los resultados de nuestra investigación con otros estudios, sin embargo, todo indica que el DBL pudiera ser atribuido una limitación neural cuando trabajan ambos miembros al mismo tiempo (Ehlers Botton \& Silveira Pinto, 2012). Aunque hace falta realizar más estudios para dilucidar el origen del DBL.

\section{Conclusiones}

Después de haber analizado los resultados se concluye que el DBL de las manifestaciones de la fuerza muscular está presente en los participantes del estudio, por igual entre hombres y mujeres. Sin embargo, es difícil comparar los resultados obtenidos en esta investigación con otros estudios debido a la utilización de diferentes instrumentos para la medición de la fuerza y las distintas características de los participantes.

\section{AplicaCiones práCticas}

Es posible que el trabajo unilateral durante el entrenamiento de fuerza mejore los resultados en el desempeño físico ya que por medio de esta forma de ejecución de los ejercicios se puede estimular en mayor medida los músculos e incrementar la carga, por lo que es importante incluir este tipo de trabajo durante la planificación del entrenamiento.

\section{Agradecimientos}

Este proyecto se llevó a cabo gracias al apoyo otorgado por el Programa para el Desarrollo del Profesional Docente (PRODEP) de la Secretaría de Educación Pública.

\section{ReFERENCIAS}

Baechle, T. R., \& Earle, R. W. (2008). Essentials of strengh traning an conditioning. Champaing, Il.: Human Kinetics.

Beurskens, R., Gollhofer, A., Muehlbauer, T., Cardinale, M., \& Granacher, U. (2015). Effects of heavy-resistance strength and balance training on unilateral and bilateral leg strength performance in old adults. PLoS One, 10(2), e0118535. Recuperado de: http://dx.doi.org/10.1371/journal.pone.0118535

Bobbert, M. F., de Graaf, W. W., Jonk, J. N., \& Casius, L. J. (2006). Explanation of the bilateral deficit in human vertical squat jumping. Journal of Applied Physiology, 100(2), 493-499. Recuperado de: http://dx.doi.org/10.1 152/japplphysiol.00637.2005

Bračič, M., Supej, M., Peharec, S., Bačić, P., \& Ćoh, M. (2010). An investigation of the infuence of bilateral deficit on the counter-movement jump performance in elite sprinters. Kinesiology, 42(1), 73-81. Recuperado de: http://d x.doi.org/10.1.1.666.323\&rep=rep1\&type $=$ pdf

Brown, L., Whitehurst, M., Gilbert, R., Findley, B., \& Buchalter, D. (1994). Effect of velocity on the bilateral deficit during dynamic knee extension and flexion exercise in female (Effet de la velocite sur le deficit bilateral lors d'exercices de flexion et extension du genou effectues par des femmes). Isokinetics \& Exercise Science, 4(4), 153-156. Recuperado de: http://dx.doi.org/ 10.3233/IES-1994-4406

Buckthorpe, M. W., Pain, M. T., \& Folland, J. P. (2013). Bilateral deficit in explosive force production is not caused by changes in agonist neural drive. PLoS One, 8(3), e57549. Recuperado de: http://dx.doi.org/10.1371/journ al.pone.0057549 
Candia-Luján, R., \& de-Paz-Fernández, J. A. (2015). Asimetría de la masa, fuerza y potencia muscular de los miembros inferiores de estudiantes universitarios. Tecnociencia, 9(1), 22-29.

Chaves, C. P. G., Guerra, C. P. C., Moura, S. R. G. d., Nicoli, A. I. V., Idemar, F., \& Simão, R. (2004). Bilateral deficit in leg flexion and extension and elbow flexion movements. Déficit bilateral nos movimentos de flexão e extensão de perna e flexão do cotovelo. Revista Brasileira de Medicina do Esporte, 10(6), 505-508. Recuperado de: http:/ /www.scielo.br/pdf/rbme/v10n6/a07v10n6

Comfort, P., \& Kasim, P. (2007). Optimizing Squat Technique. Strength \& Conditioning Journal, 29(6), 10-13. Recuperado de: http://dx.doi.org/10.1519/00126548-200712000-00001

Cronin, J. B., Hing, R. D., \& McNair, P. J. (2004). Reliability and validity of a linear position transducer for measuring jump performance. Journal of Strength \& Conditioning Research, 18(3), 590-593. Recuperado de: http://dx.do i.org/10.1519/00124278-200408000-00035

Cureton, K. J., Collins, M. A., Hill, D. W., \& McElhannon, F. M. (1988). Muscle hypertrophy in men and women. Medicine \& Science in Sports \& Exercise, 20(4), 338-344. Recuperado de: http://dx.doi.org/10.1249/0000576 8-198808000-00003

de Souza Bezerra, E., da Silva Bezerra, É. Q., Simão, R., Dias, I., \& Figueiredo, T. (2012). Déficit bilateral de força pósreconstrução do ligamento cruzado anterior. / Bilateral deficit of strength after reconstruction of the anterior cruciate ligament. Brazilian Journal of Kineanthropometry \& Human Performance, 14(1), 93-100. Recuperado de: http://dx.doi.org/10.5007/1980-0037.2012v14n1p93

Dickin, D. C., \& Too, D. (2006). Effects of movement velocity and maximal concentric and eccentric actions on the bilateral deficit. Research Quarterly for Exercise \& Sport, 77(3), 296-303. Recuperado de: http://dx.doi.org/10. 1080/02701367.2006.10599363

Dunstheimer, D., Hebestreit, H., Staschen, B., Strassburg, H. M., \& Jeschke, R. (2001). Bilateral deficit during shortterm, high-intensity cycle ergometry in girls and boys. / Deficit bilateral au cours d ' un cycle ergometrique a cours terme et de haute intensite chez les garcons et les filles. European Journal of Applied Physiology, 84(6), 557-561. Recuperado de: http://dx.doi.org/10.1007/s004210100406

Ebben, W. P., Flanagan, E., \& Jensen, R. L. (2009). Bilateral facilitation and laterality during the contermovement jump. Perceptual and Motor Skills, 108(1), 251-258. Recuperado de: http://dx.doi.org/10.2466/pms.108.1.25 $1-258$

Ehlers Botton, C., \& Silveira Pinto, R. (2012). Déficit bilateral: origem, mecanismos e implicações para o treino de força. / Bilateral deficit: origins, mechanisms, and implications for strength training. Brazilian Journal of Kineanthropometry \& Human Performance, 14(6), 749-761. Recuperado de: http://dx.doi.org/10.5007/1980 $-0037.2012 \mathrm{v} 14 \mathrm{n} 6 \mathrm{p} 749$

Hernandez, J. P., Nelson-Whalen, N. L., Franke, W. D., \& McLean, S. P. (2003). Bilateral index expressions and iEMG activity in older versus young adults. The Journals of Gerontology Series A: Biological Sciences and Medical Sciences, 58(6), M536-M541. Recuperado de: http://dx.doi.org/10.1093/gerona/58.6.M536

Howard, J. D., \& Enoka, R. M. (1991). Maximum bilateral contractions are modified by neurally mediated interlimb effects. Journal of Applied Physiology, 70(1), 306-316.

Jakobi, J. M., \& Chilibeck, P.D. (2001). Bilateral and unilateral contractions: possible differences in maximal voluntary force. Canadian Journal of Applied Physiology, 26(1), 12-33. Recuperado de: http://dx.doi.org/10.1139/ h01-002

Kawamori, N., \& Haff, G. G. (2004). The optimal training load for the development of muscular power. Journal of Strength \& Conditioning Research, 18(3), 675-684. Recuperado de: http://dx.doi.org/10.1519/00124278-200 408000-00051

Kuruganti, U., Murphy, T., \& Pardy, T. (2011). Bilateral deficit phenomenon and the role of antagonist muscle activity during maximal isometric knee extensions in young, athletic men. European Journal of Applied Physiology, 111(7), 1533-1539. DOI 10.1007/s00421-010-1752-8. Recuperado de: http://dx.doi.org/10.1007/s00421-01 0-1752-8 
Kuruganti, U., \& Seaman, K. (2006). The bilateral leg strength deficit is present in old, young and adolescent females during isokinetic knee extension and flexion. European Journal of Applied Physiology, 97(3), 322-326. Recuperado de: http://dx.doi.org/10.1007/s00421-006-0188-7

MacArdle, W., Katch, FI. \& Katch, VL. (2010). Exercise Physiology. Nutrition, energy and human performance. Philadelphia; Lippincott Williams \& Wilkins.

MacDonald, M., Losier, D., Chester, V. L., \& Kuruganti, U. (2014). Comparison of bilateral and unilateral contractions between swimmers and nonathletes during leg press and hand grip exercises. Applied Physiology, Nutrition \& Metabolism, 39(11), 1245-1249. Recuperado de: http://dx.doi.org/10.1139/apnm-2014-0040

Magnus, C. R. A., \& Farthing, J. P. (2008). Greater bilateral deficit in leg press than in handgrip exercise might be linked to differences in postural stability requirements. Applied Physiology, Nutrition \& Metabolism, 33(6), 1132-1139. Recuperado de: http://dx.doi.org/10.1139/H08-101

Matkowski, B., Martin, A., \& Lepers, R. (2011). Comparison of maximal unilateral versus bilateral voluntary contraction force. European Journal of Applied Physiology, 111(8), 1571-1578. Recuperado de: http:// dx.doi.org/ 10.1007/s00421-010-1775-1

Maughan, R. J., Watson, J. S., \& Weir, J. (1983). Strength and cross-sectional area of human skeletal muscle. Journal of Physiology, 338, 37-49.

Monteiro, W. D., \& Simão, R. (2006). Is there bilateral deficit in the practice of 10RM in arm and leg exercises?Existe déficit bilateral na realização de 10RM em exercícios de braços e pernas? Revista Brasileira de Medicina do Esporte, 12(3), 115-118. Recuperado de: http://dx.doi.org/10.1590/S1517-86922006000300001

Nazário-de-Rezende, F., Haddad, E. G., Sousa, G. d. C., de Agostini, G. G., Dias Nunes, J. E., \& Marocolo Jr, M. (2012). Bilateral deficit in multiarticular exercise for upper extremities. Revista Brasileira de Medicina do Esporte, 18(6), 385-389. Recuperado de: http://dx.doi.org/10.1590/S1517-86922012000600008

Nijem, R. M., \& Galpin, A. J. (2014). Unilateral versus bilateral exercise and the role of the bilateral force deficit. Strength \& Conditioning Journal, 36(5), 113-118. Recuperado de: http://dx.doi.org/10.1519/SSC.000000000 0000085

Owings, T. M., \& Grabiner, M. D. (1998). Normally aging older adults demonstrate the bilateral deficit during ramp and hold contractions. The Journals of Gerontology Series A: Biological Sciences and Medical Sciences, 53A(6), B425-B429. Recuperado de: http://dx.doi.org/10.1093/gerona/53A.6.B425

Pain, M. T. G. (2014). Considerations for single and double leg drop jumps: bilateral deficit, standardizing drop Height, and equalizing training load. Journal of Applied Biomechanics 30(6), 722-727. Recuperado de: http:/ /dx.doi.org/10.1123/jab.2014-0035

Schantz, P. G., Moritani, T., Karlson, E., Johansson, E., \& Lundh, A. (1989). Maximal voluntary force of bilateral and unilateral leg extension. Acta Physiologica Scandinavica, 136(2), 185-192. Recuperado de: http://dx.doi.org/10 $.1111 / j .1748-1716.1989 . t b 08651 . x$

Siegel, J. A., Gilders, R. M., Staron, R. S., \& Hagerman, F. C. (2002). Human muscle power output during upper- and lower-body exercises. Journal of Strength \& Conditioning Research, 16(2), 173-178. Recuperado de: http://dx.d oi.org/10.1519/00124278-200205000-00002

Simao, R., Lemos, A., Viveiros, L. E., Chaves, C. P. G., \& D, P. M. (2003). Maximum muscular strength in unilateral versus bilateral leg extension. Revista Brasileira de Fisiologia do Exercicio, 2, 29-39.

Teixeira, A. L. S., Narciso, J. C. A., Taroco Salomão, I., \& Dias, M. R. C. (2013). Bilateral deficit in maximal isometric knee extension in trained men. Journal of Exercise Physiology Online, 16(1), 28-35.

Van Dieen, J. H., Ogita, F., \& De Haan, A. (2003). Reduced neural drive in bilateral exertions: a performance-limiting factor? / La baisse de l' influx nerveux lors des efforts bilateraux est-elle un facteur de limitation des performances? Medicine \& Science in Sports \& Exercise, 35(1), 111-118. Recuperado de: http://dx.doi.org/10.1097/0000576 8-200301000-00018

Veligekas, P., \& Bogdanis, G. C. (2013). Bilateral deficit in vertical jumping in pre-pubertal boys and girls. Journal of Physical Education \& Sport, 13(1), 120-126. 
Ramón Candia-Luján, et al. Déficit bilateral de las manifestaciones de la fuerza muscular de las e...

Willardson, J. M., \& Tudor-Locke, C. (2005). Survival of the strongest: A brief review examining the association between muscular fitness and mortality. Journal of Strength \& Conditioning Journal, 27(3), 80-85. Recuperado de: http://dx.doi.org/10.1519/1533-4295(2005)027[0080:sotsab]2.0.co;2

Recepción: 03 Marzo 2017

Aprobación: 15 Octubre 2017

La aceptación de colaboraciones por parte de la revista implica la cesión no exclusiva de los derechos patrimoniales de los autores a favor del editor, quien permite la reutilización, luego de su edición (postprint), bajo Licencia Creative Commons Atribución-NoComercial-CompartirIgual 4.0 Internacional (https:// creativecommons.org/licenses/by-nc-sa/4.0/). CC BY-SA 